\begin{tabular}{|c|l|}
\hline Title & $\begin{array}{l}\text { Photochemical hy drogen evolution from aqueous triethanol amine solutions sensitized by binaphthol-modified } \\
\text { titanium(IV) oxide under visible light irradiation }\end{array}$ \\
\hline Author(s) & Ikeda, Shigeru; A be, Chigusa; Torimoto, Tsukasa; Ohtani, Bunsho \\
\hline Citation & $\begin{array}{l}\text { Journal of Photochemistry and Photobiology A Chemistry, 160(1-2), 61-67 } \\
\text { https:/doi.org/10.1016/S1010-6030/03)00222-3 }\end{array}$ \\
\hline Issue Date & $2003-08$-07 \\
\hline Doc URL & http://hdl.handle.net/2115/14647 \\
\hline Type & article (author version) \\
\hline File Information & JPAP2003-160-1-2.pdf \\
\hline
\end{tabular}

Instructions for use 


\title{
Photochemical Hydrogen Evolution from Aqueous Triethanolamine Solutions Sensitized by Binaphthol-Modified Titanium(IV) Oxide under Visible-Light Irradiation
}

Shigeru Ikeda, ${ }^{\mathrm{a}, \mathrm{b}}$ Chigusa Abe, ${ }^{\mathrm{b}}$ Tsukasa Torimoto, ${ }^{\mathrm{a}, \mathrm{b}}$ and Bunsho Ohtani ${ }^{*}$,a,

a Catalysis Research Center, Hokkaido University, Sapporo 060-0811, Japan

b Graduate School of Environmental Earth Science, Hokkaido University, Sapporo 060-0810, Japan

Corresponding author. Tel: +81-11-706-3673; fax: +81-11-706-4925. E-mail address: ohtani@cat.hokudai.ac.jp (B. Ohtani).

\begin{abstract}
Surface modification of titanium(IV) oxide $\left(\mathrm{TiO}_{2}\right)$ powders with 1,1'-binaphthalene-2,2'-diol (bn $\left.(\mathrm{OH})_{2}\right)$, having two phenolic hydroxyls and atropisomeric chirality, in refluxed ethanol led to the formation of a deep yellow-colored surface complex, giving visible-light absorption at a wavelength below ca. 550-600 nm. Quantitative analyses of the surface complex revealed that absorption intensity at $450 \mathrm{~nm}$ in diffuse reflection measurement was almost proportional to its amount and that its amount depended strongly on the amount of surface hydroxyl groups of $\mathrm{TiO}_{2}$ but not on the crystal structure of $\mathrm{TiO}_{2}$, anatase and rutile. The bn $(\mathrm{OH})_{2}$ complexation was also applied to platinized $\mathrm{TiO}_{2}$ to drive photocatalytic molecular hydrogen $\left(\mathrm{H}_{2}\right)$ evolution from
\end{abstract}


deaerated triethanolamine solutions under visible-light irradiation at a wavelength even longer than $540 \mathrm{~nm}$. The photonic efficiency of the photocatalytic reaction at $450 \mathrm{~nm}$ was estimated to be $0.02 \%$ for bn $(\mathrm{OH})_{2}$-modified $\mathrm{TiO}_{2}$ (JRC-TIO-3) loaded with 0.1 wt\% of platinum. On the basis of these experimental results, the reaction mechanism, which involves visible-light excitation of the surface complex, injection of electrons from the complex to $\mathrm{TiO}_{2}$, and migration of the electrons to platinum deposits, where reduction of $\mathrm{H}^{+}$takes place to give $\mathrm{H}_{2}$, was elucidated.

Keywords: titanium(IV) oxide; 1,1'-binaphthalene-2,2'-diol; surface complex; visible light; sensitization; photocatalytic reaction

\section{Introduction}

It is generally of great importance to control the activity of $\mathrm{TiO}_{2}$ photocatalysts by modification of their bulk and/or surface properties in compliance with different needs $[1,2]$. One of the intriguing subjects in both academic research and industrial research is development of new $\mathrm{TiO}_{2}$-photocatalytic systems with enhanced activities under visible-light irradiation compared with the simply $\mathrm{TiO}_{2}$-photocatalyzed processes, since bare $\mathrm{TiO}_{2}$ can absorb light in the ultraviolet range, which corresponds to only ca. 3\% of the energy of sunlight. Several strategies and methods for modifying the optical properties of $\mathrm{TiO}_{2}$ particles, e.g., doping of a transition metal [3,4], plasma treatments [5] and nitrogen doping [6,7], have been proposed, and photoactivity under visible-light irradiation has been proved in some limited reactions. Much interest has also been shown in surface modification of $\mathrm{TiO}_{2}$ with transition metal complexes and/or organic dyes for construction of dye-sensitized photocatalytic systems that work under visible-light irradiation [8,9] as well as for improvement of the efficiency of dye-sensitized solar cells [10-12]. 
On the other hand, in order to clarify the reaction mechanism of the photocatalytic reaction of organic molecules, structural characteristics of these compounds adsorbed on $\mathrm{TiO}_{2}$ surface have been extensively investigated using various spectroscopic techniques [13-19]. In the course of these studies, it was proved that colorless aromatic compounds having phenolic hydroxyls such as catechol and salicylic acid react with $\mathrm{TiO}_{2}$ particles to form colored surface complexes [15-19]. Thus, it is expected that a novel visible-light-induced photocatalytic system can be developed by using the photoabsorption of surface complexes on $\mathrm{TiO}_{2}$ particles. Recently, we have demonstrated that 1,1'-binaphthalene-2,2'-diol (bn $\left.(\mathrm{OH})_{2}\right)$, having two phenolic hydroxyls and atropisomeric chirality, reacts with $\mathrm{TiO}_{2}$ and gives a colored surface complex, which works as catalyst for water reduction in the presence of a sacrificial donor under visible-light irradiation [20].

In this paper, we describe in detail the structural characteristics of the surface $\mathrm{TiO}_{2}$-bn $(\mathrm{OH})_{2}$ complex and some mechanistic aspects of visible light-induced photoactivity.

\section{Experimental}

\subsection{Materials}

Several kinds of titanium oxide $\left(\mathrm{TiO}_{2}\right)$ powders, those from commercial sources (Degussa P25, Degussa F281, Ishihara ST-01, Ishihara CR-EL, Hombikat UV-100, Showa Denko F-6, and Idemitsu UF), reference catalysts supplied from the Catalysis Society of Japan (TIO-2 and TIO-3), and powders synthesized by the HyCOM method [21,22] followed by calcination at $573 \mathrm{~K}$ under $\mathrm{O}_{2}$, were used. The properties of these $\mathrm{TiO}_{2}$ powders are summarized in Table 1. Ethanol (Wako), methanol (Wako), triethanolamine (2,2',2'’-nitrilotrisethanol, TEOA; Wako), chloroplatinic acid $\left(\mathrm{H}_{2} \mathrm{PtCl}_{6} \cdot 6 \mathrm{H}_{2} \mathrm{O}\right.$, Wako), and 
1,1'-binaphthalene-2,2'-diol (bn $(\mathrm{OH})_{2}$, racemate; Aldrich), were used without further purification.

\subsection{Preparation of bn $(\mathrm{OH})_{2}$-adsorbed $\mathrm{TiO}_{2}$}

In a typical experiment, $0.5 \mathrm{~g}$ of $\mathrm{TiO}_{2}$ powder was suspended in an ethanolic solution of bn(OH) $\left.(0.14 \mathrm{~mol} \mathrm{dm})^{-3}, 25 \mathrm{~cm}^{3}\right)$, and the resulting suspension was refluxed for $8 \mathrm{~h}$ under an argon (Ar) atmosphere. The solid was collected by centrifugation (ca. $3500 \mathrm{rpm}$ ), washed twice with ca. $10 \mathrm{~cm}^{3}$ of ethanol, and dried under reduced pressure at ambient temperature. Ultraviolet and visible-light diffuse reflection (DR) spectra were obtained using a Hamamatsu Photonics C7473-6 Photonic Multi-Channel Analyzer, and the data were converted to Kubelka-Munk (KM) function. Based on the contents of carbon and ash components quantified by elemental analysis, the amount of adsorbed $\mathrm{bn}(\mathrm{OH})_{2}$ was estimated with assumption that the remaining ash was only composed of $\mathrm{TiO}_{2}$. Since the carbon components in most of the samples were less than $2 \mathrm{wt} \%$ and the elemental analysis of organic compounds includes less than $0.3 \%$ of error, estimated amounts may include a large quantity of error. However, we could confirm reproducibility within 10\% on some selected samples. Platinized $\mathrm{TiO}_{2}$ powder, prepared by photoplatinization in deaerated aqueous methanol solution containing $\mathrm{H}_{2} \mathrm{PtCl}_{6} \cdot 6 \mathrm{H}_{2} \mathrm{O}$ [24], was also modified with bn $(\mathrm{OH})_{2}$ by the same procedure as that used for unplatinized $\mathrm{TiO}_{2}$ powder.

\subsection{Photoirradiation and product analysis}

The bn(OH) $)_{2}$-modified or bare $\mathrm{TiO}_{2}$ powder $(50 \mathrm{mg})$ and an aqueous TEOA (10 vol\%) or methanol (50 vol\%) solution $\left(5 \mathrm{~cm}^{3}\right)$ were placed in a glass tube (transparent at $>$ $300 \mathrm{~nm}, 18 \mathrm{~mm}$ in diameter and $180 \mathrm{~mm}$ in length), and the suspensions were photoirradiated under $\mathrm{Ar}$ at $298 \mathrm{~K}$ with vigorous magnetic stirring (1000 rpm). Photoirradiation was performed using a high-pressure mercury arc (Eiko-sha, $400 \mathrm{~W}$ ) or a 
xenon arc (Ushio UXR-300DU, $300 \mathrm{~W}$ ) with a Y-44, Y-50, O-55, or O-59 optical filter (Asahi Techno Glass) to cut out light of wavelength shorter than 430, 490, 540 or $580 \mathrm{~nm}$, respectively. Liberated hydrogen $\left(\mathrm{H}_{2}\right)$ was analyzed using a gas chromatograph (GC, Shimadzu GC-8A) equipped with an MS-5A column (GL Sciences) and a TCD detector. Photonic efficiency, a molar ratio of product (twice the amount of $\mathrm{H}_{2}$ ) to incident photons, was estimated at $450 \mathrm{~nm}$ using a JASCO CT-101T monochromator equipped with a xenon arc. The incident photon flux was estimated using a Molectron POWER MAX5200 laser power meter.

\section{Results and Discussion}

\subsection{Structure of $\mathrm{TiO}_{2}$ modified with $b n(\mathrm{OH})_{2}$}

Figure 1 shows ultraviolet and visible DR spectra of Degussa P25, TIO-3, and $\mathrm{TiO}_{2}$ powders treated with an ethanolic bn $(\mathrm{OH})_{2}$ solution. Untreated P25 and TIO-3 gave absorption in the ultraviolet region below ca. $400 \mathrm{~nm}$ and $410 \mathrm{~nm}$, respectively, corresponding to their band-gap photoabsorption. The gaps were reported to be ca. $3.0 \mathrm{eV}$ $(410 \mathrm{~nm})$ for rutile (TIO-3) and $3.2 \mathrm{eV}(390 \mathrm{~nm})$ for anatase [25], and P25, a mixture of anatase and rutile, might give an intermediate spectrum. Treatment of the colorless compound bn $(\mathrm{OH})_{2}$ induced striking changes in the spectral features of these $\mathrm{TiO}_{2}$ powders: the color of the powders became deep yellow after bn $(\mathrm{OH})_{2}$ treatment, giving visible-light absorption below ca. 550-600 $\mathrm{nm}$. Due to the overlap of the fundamental band gap photoabsorption of $\mathrm{TiO}_{2}$, the exact position of the peak, if present, of newly appearing visible-light absorption could not be determined. However, the shapes in the visible range and the absorption edge positions of the two bn $(\mathrm{OH})_{2}$-treated $\mathrm{TiO}_{2}$ powders were quite similar; there seemed to be no significant dependence on the crystal form, anatase and rutile of $\mathrm{TiO}_{2}$. 
To identify the surface species, the amounts of bn $(\mathrm{OH})_{2}$ attached to several $\mathrm{TiO}_{2}$ powders were quantified by elemental analysis of carbon and ash components. Figure 2 shows plots of absorption intensity at $450 \mathrm{~nm}$ of bn(OH$)_{2}$-treated $\mathrm{TiO}_{2}$ powder $\left(\mathrm{I}_{450}\right)$ and its BET surface area (S) as a function of the amount of adsorbed $b n(\mathrm{OH})_{2}$ per unit weight of $\mathrm{TiO}_{2}\left(\mathrm{M}_{\mathrm{bn}}\right)$. It is clear from this figure that there is an almost linear relation between $\mathrm{M}_{\mathrm{bn}}$ and $\mathrm{I}_{450}$ of several $\mathrm{TiO}_{2}$ powders. This suggests that the $\mathrm{bn}(\mathrm{OH})_{2}$ species adsorbed on $\mathrm{TiO}_{2}$ give visible light absorption, independent of the kind of bare $\mathrm{TiO}_{2}$ powder. Since $\mathrm{I}_{450}$ was measured in KM function which contains a term of light scattering as well as that of absorption and thus it depends on the light scattering properties of bare $\mathrm{TiO}_{2}$ powders, deviation of a plot (g) higher from a linear line might be caused by the scattering properties of these $\mathrm{TiO}_{2}$ powders (consisting of amorphous) which seem a different from the ordinary crystalline powders. It is also noted that a plot (e) seems to slightly deviate lower from a linear line. Although there is no clear basis at present, this might be due to the presence of another type of a surface complex on the sample (e), having lower absorption coefficient than that on other ordinal $\mathrm{TiO}_{2}$ powders (see below). A linear relationship was also observed between $\mathrm{M}_{\mathrm{bn}}$ and $\mathrm{S}$ for all $\mathrm{TiO}_{2}$ powders except for the amorphous $\mathrm{TiO}_{2}$ (Idemitsu UF), which showed a relatively high density of $\mathrm{bn}(\mathrm{OH})_{2}$ adsorption and as is discussed in the following section.

As already reported by several workers [15-19], white $\mathrm{TiO}_{2}$ particles and colloids turn yellow upon immersion in a solution containing phenols such as salicylic acid and catechol. The development of the yellow color is a clear indication of the formation of surface $\mathrm{TiO}_{2}$-salicylate and $\mathrm{TiO}_{2}$-catecholate complexes, and the absorption bands in the visible range were assigned to intramolecular ligand-to-metal charge transfer transition $[18,19]$. Thus, the observed visible-light absorption in the present experiment is attributable to the formation of a surface $\mathrm{TiO}_{2}-\mathrm{bn}(\mathrm{OH})_{2}$ complex. According to previous reports on $\mathrm{TiO}_{2}$-salicylate and $\mathrm{TiO}_{2}$-catecholate surface complexes [16-19], there appears to be two different structures of the complex. One structure is formed by chelation of a surface titanium atom with two hydroxyl groups in a bn $(\mathrm{OH})_{2}$ molecule (“chelation”, a 1:2 
adduct) and the other is formed by binding of each phenolic hydroxyl group in bn(OH $)_{2}$ to the surface titanium atom (“esterification”, a 2:2 adduct), both of which are induced by intermolecular dehydration of surface and phenolic hydroxyls, as shown in Scheme 1. Although there is no experimental method for discriminating these two possible structures of the surface complex in the samples, the fact that a yellow-colored powder giving a similar absorption spectrum in the visible light range below ca. $550 \mathrm{~nm}$ was also obtained by the reaction of $\mathrm{TiO}_{2}$ with 2-naphthol, which has only one phenolic hydroxyl group (data not shown), suggested that the latter structure ("esterification") could interpret the present results of bn(OH) , but did not exclude the former structure (“chelation”). Further studies for clarification of the structure are now underway.

Previous studies on the quantification of surface hydroxyls (Ti-OH) on $\mathrm{TiO}_{2}$ by sodium hydroxide titration [26] have shown that the density of Ti-OH is almost constant, ca. 2.5 hydroxyls $\mathrm{nm}^{-2}$, among several polycrystalline $\mathrm{TiO}_{2}$ powders except for amorphous samples, which have a relatively high density of Ti-OH, ca. 16 hydroxyls $\mathrm{nm}^{-2}$ [27]. Assuming surface complexation through dehydration between Ti-OH with the hydroxyl groups in bn $(\mathrm{OH})_{2}$ as discussed above and uniform and similar chemical properties of Ti-OH in $\mathrm{TiO}_{2}$ samples, a linear relationship between $\mathrm{M}_{\mathrm{bn}}$ and $\mathrm{S}$, as shown in Fig. 2, seems quite reasonable for the samples of $\mathrm{TiO}_{2}$ having almost the same Ti-OH surface density. Deviation of the Idemistu UF ((g) in Fig. 2) is thought to be due to its higher density of surface Ti-OH. From the reciprocal slope of Fig. 2 (lower), the surface density of $b n(O H)_{2}$ on the ordinary polycrystalline samples was estimated to be $0.8 \mu \mathrm{mol} \mathrm{m} \mathrm{m}^{-2}$ or 0.48 molecules $\mathrm{nm}^{-2}$. The density was ca. $1 / 5$ of the above estimated Ti-OH density, and approximately half (or little less) of $\mathrm{Ti}-\mathrm{OH}$ was used for surface complexation when the 2:2 adduct was presumed. This estimation of surface coverage is consistent with the estimated coverage, half or a little less, using the cross-sectional area of a bn(OH $)_{2}$ molecule (ca. $1 \mathrm{~nm}^{2}$ ) in the surface structure shown in Scheme 1. It is reasonable to assume that the loosely packed arrangement of bn $(\mathrm{OH})_{2}$ is caused by its large molecular size and a mismatching of the Ti-OH spatial arrangement to anchor bn $(\mathrm{OH})_{2}$. On the other 
hand, the higher density of $\operatorname{bn}(\mathrm{OH})_{2}$ on Idemitsu UF was attributed to the sufficiently high density of Ti-OH to allow close packing of $\mathrm{bn}(\mathrm{OH})_{2}$ on the surface. The estimated density of bn $(\mathrm{OH})_{2}$ on UF, ca. 2.8 molecules $\mathrm{nm}^{-2}$, was three-times larger than that estimated from the cross-sectional area. This might be caused, at least partly, by underestimation of its BET surface area; surface adsorption of nitrogen was measured after evacuation at $383 \mathrm{~K}$, which was $>100 \mathrm{~K}$ lower than that for the other $\mathrm{TiO}_{2}$ samples, to minimize the thermal effect on its structure [28] and at the same time to allow water to remain on the surface.

Thus, a part of the surface of bn $(\mathrm{OH})_{2}$-modified $\mathrm{TiO}_{2}$ particles remains uncovered with $\mathrm{bn}(\mathrm{OH})_{2}$, and it is therefore expected that photoinduced reactions can be driven by bn $(\mathrm{OH})_{2}$-modified $\mathrm{TiO}_{2}$ with photoabsorption by surface complexes and with redox reactions by photoexcited electrons (or holes) on the unmodified bare surface.

\subsection{Photoreactivity of platinized $\mathrm{TiO}_{2}$ modified with $b n(\mathrm{OH})_{2}$}

Photoinduced reduction of water to produce $\mathrm{H}_{2}$ from an aqueous solution containing a sacrificial electron donor, methanol or TEOA, was conducted as a test reaction of photoactivity of the $\mathrm{bn}(\mathrm{OH})_{2}$-modified $\mathrm{TiO}_{2}$. Preliminary experiments revealed the requirement of platinization to make active sites on the surface for $\mathrm{H}_{2}$ production. Therefore, before the photoactivity test, a small amount of platinum (Pt) particles was deposited on $\mathrm{TiO}_{2}(0.05-2 \mathrm{wt} \%$, corresponding to ca. $0.2-5 \%$ of surface coverage [29]) followed by modification with $\operatorname{bn}(\mathrm{OH})_{2}$. Consistent with this small coverage of $\mathrm{Pt}$ particles on $\mathrm{TiO}_{2}, \mathrm{M}_{\mathrm{bn}}$ on platinized (2 wt\%) TIO-3 (40 $\mu$ mol g ${ }^{-1}$ ) was comparable or even larger than that on bare TIO-3 (32 $\left.\mu \mathrm{mol} \mathrm{g}^{-1}\right)$. One possible explanation for this small increment is attachment of $\mathrm{bn}(\mathrm{OH})_{2}$ to the surface of Pt particles. We tried to obtain the absorption spectrum of bn $(\mathrm{OH})_{2}$ complex on platinized $\mathrm{TiO}_{2}$ but failed due to interference by gray-colored Pt particles. Another possible explanation is an increase in Ti-OH during the Pt photodeposition process; the formation of Ti-OH induced by band-gap excitation of $\mathrm{TiO}_{2}$ has been reported in relation with a phenomenon of photoinduced super 
hydrophilicity [30].

Figure 3 shows $\mathrm{H}_{2}$ liberation from aqueous methanol and TEOA solutions under ultraviolet light irradiation (> $300 \mathrm{~nm}$ ) over bn $(\mathrm{OH})_{2}$-modified and unmodified $\mathrm{TiO}_{2}$ (TIO-3). In all the experimental runs, a linear increase in the molar amount of $\mathrm{H}_{2}$ was observed. Due to the lower solubility of TEOA in water, the concentrations of these two electron donors were different (50 and $10 \mathrm{vol} \%$ for methanol and TEOA, respectively), and $\mathrm{pH}$ of an aqueous TEOA solution (originally ca. 11) was not adjusted to be neutral. The electron-donating ability, therefore, can not be discussed on the basis of these results. It is notable that $\mathrm{bn}(\mathrm{OH})_{2}$-modified $\mathrm{TiO}_{2}$ showed almost the same or an even slightly higher rate than that of unmodified $\mathrm{TiO}_{2}$ in the aqueous methanol suspension, suggesting negligible or at most only partial coverage of the surface of Pt particles by bn $(\mathrm{OH})_{2}$. On the other hand, when TEOA was used as a sacrificial electron donor, the $\mathrm{H}_{2}$-liberation rate was halved by the $\mathrm{bn}(\mathrm{OH})_{2}$ modification. It was presumed that access of TEOA, the size of which is much larger than that of methanol, to the bare (not covered with $b(\mathrm{OH})_{2}$ ) surface was restricted. In a $\mathrm{TiO}_{2}$-photocatalyzed reaction using photoexcited electrons and positive holes in $\mathrm{TiO}_{2}$, adsorption of a reaction substrate such as TEOA on bare $\mathrm{TiO}_{2}$ is required.

Figure 4 shows the time courses of $\mathrm{H}_{2}$ liberation from unmodified and bn $(\mathrm{OH})_{2}$-modified $\mathrm{TiO}_{2}$ (TIO-3) from an aqueous TEOA solution under visible-light irradiation (> $430 \mathrm{~nm}) . \mathrm{H}_{2}$ liberation from the $\mathrm{bn}(\mathrm{OH})_{2}$-modified $\mathrm{TiO}_{2}$ suspension was observed, while, as predicted from the negligible photoabsorption of bare $\mathrm{TiO}_{2}$ in the visible region (Fig. 1), no appreciable liberation of $\mathrm{H}_{2}$ from unmodified $\mathrm{TiO}_{2}$ was detected [20]. The total amount of evolved $\mathrm{H}_{2}$ after $80 \mathrm{~h}$ of irradiation reached more than $40 \mu \mathrm{mol}$. Since the amount of $\mathrm{bn}(\mathrm{OH})_{2}$ on $50 \mathrm{mg}$ of TIO-3 was estimated to be $2 \mu \mathrm{mol}$ (corresponding to $24 \mu \mathrm{mol}$ of $\mathrm{H}$ atoms based on the assumption of the structure shown in Scheme 1), the total $\mathrm{H}_{2}$ yield was more than 20-times greater than the molar amount of bn $(\mathrm{OH})_{2}$, indicating that the photoinduced reaction proceeded catalytically. It is also notable that the rate of $\mathrm{H}_{2}$ production decreased gradually. This may be due to 
decomposition or leaching of the surface complex during the reaction. At present, however, there is no experimental evidence to elucidate the decrease of adsorbed bn $(\mathrm{OH})_{2}$, mainly because of the interference by Pt deposits, which causes greatly reduced diffuse reflection and difficulty in evaluating DR spectra with high accuracy and reproducibility. In the second run of irradiation after Ar bubbling to purge liberated $\mathrm{H}_{2}$ and other volatile compounds such as ammonia, the initial rate $\left(0.65 \mu \mathrm{mol} \mathrm{h}{ }^{-1}\right)$ was less than half of that in the first run $\left(1.7 \mu \mathrm{mol} \mathrm{h}^{-1}\right)$ but was more than $30 \%$ higher than the rate in the final stage of the first run $\left(0.48 \mu \mathrm{mol} \mathrm{h}{ }^{-1}\right)$. These facts indicate that accumulation of a volatile by-product(s) accounts at least partly for the deactivation during the course of irradiation. Under visible-light irradiation, negligible $\mathrm{H}_{2}$ liberation was observed when methanol was used as a sacrificial electron donor instead of TEOA even at high $\mathrm{pH}$ (similar to the TEOA solution), though methanol worked efficiently when $\mathrm{TiO}_{2}$ was photoexcited under ultraviolet-light irradiation (see Fig. 3).

Figure 5 shows dependence of the rate of $\mathrm{H}_{2}$ liberation on the wavelength of irradiated light for bn(OH)$)_{2}$-modified $\mathrm{TiO}_{2}$ (TIO-3) loaded with 0.5 wt\% of Pt. The $\mathrm{H}_{2}$ liberation required irradiation at a wavelength of $<580 \mathrm{~nm}$; no $\mathrm{H}_{2}$ was produced under photoirradiation at a wavelength longer than $580 \mathrm{~nm}$. This wavelength dependence corresponded to photoabsorption of $\mathrm{TiO}_{2}-\mathrm{bn}(\mathrm{OH})_{2}$ complexes shown in Fig. 1, i.e., the observed visible light-induced $\mathrm{H}_{2}$ liberation was derived from photoexcitation of the surface bn $(\mathrm{OH})_{2}$ complex on $\mathrm{TiO}_{2}$.

Figure 6 shows dependence of the rate of $\mathrm{H}_{2}$ liberation on the amount of $\mathrm{Pt}$ deposits for bn(OH)$)_{2}$-modified $\mathrm{TiO}_{2}$ (TIO-3). No $\mathrm{H}_{2}$ liberation was observed in the absence of loaded Pt. The highest activity of ca. $7 \mu \mathrm{mol} \mathrm{h}^{-1}$ was observed when $0.1 \mathrm{wt} \%$ of Pt was deposited on $\mathrm{TiO}_{2}$ (TIO-3), and the photonic efficiency of this powder was estimated to be ca. $0.02 \%$ [20]. Further loading reduced the activity, presumably due to reduction of photoabsorption by the $\mathrm{bn}(\mathrm{OH})_{2}$ surface complex and/or enhanced deactivation of the photoexcited complex. A previous study on characterization of $\mathrm{Pt}$ deposits on $\mathrm{P} 25 \mathrm{TiO}_{2}$ powder [29] revealed that the optimum Pt deposition for P25 (0.1 
wt\%), the surface area of which is similar to that of TIO-3, corresponds to the minimum amount of Pt loading required to distribute at least one Pt deposit (ca. $2 \mathrm{~nm}$ ) on each $\mathrm{TiO}_{2}$ particle. The Pt deposits act as active sites for $\mathrm{H}_{2}$ liberation through reduction of $\mathrm{H}^{+}$with photoexcited electrons of the surface bn $(\mathrm{OH})_{2}$ complex that have migrated presumably via $\mathrm{TiO}_{2}$.

The $\mathrm{TiO}_{2}$ powders such as Ishihara ST-01 and HyCOM, both of which consist of anatase crystallites, also produced $\mathrm{H}_{2}$ under the visible-light irradiation at $>430 \mathrm{~nm}$. The rates of $\mathrm{H}_{2}$ liberation were $0.5 \mu \mathrm{mol} \mathrm{h}{ }^{-1}$ for ST-01 with $2 \mathrm{wt} \%$ of Pt and $3.7 \mu \mathrm{mol} \mathrm{h}^{-1}$ for HyCOM with $1 \mathrm{wt} \%$ of Pt, respectively [20]. Although the activity of these samples is thought to depend on the amount of Pt loading, similar to that in Fig. 6, and although the amount of Pt deposits for these samples has not been optimized, a comparison of the trends in $\mathrm{H}_{2}$ liberation rates for the three samples with those of $\mathrm{M}_{\mathrm{bn}}$ (Fig. 2) indicates that there are no simple proportional relations between them. The relatively lower activity of ST-01, consisting of less crystallized anatase particles, i.e., having a larger number of defects, in spite of larger $\mathrm{M}_{\mathrm{bn}}$, suggested the importance of crystallinity of $\mathrm{TiO}_{2}$, as has been reported for the photocatalytic activity of platinized $\mathrm{TiO}_{2}$ for dehydrogenation of alcohol [22]. In the presumed mechanism, as discussed in the following section, photoexcited electrons in the surface bn(OH) 2 complex are injected into $\mathrm{TiO}_{2}$ and migrate in $\mathrm{TiO}_{2}$ to reach the Pt deposit, and the crystallinity governs the efficiency of the electron migration. Anyway, highly active photocatalytic systems are to be constructed by selection of suitable $\mathrm{TiO}_{2}$.

As suggested in the preliminary paper [20], visible light-induced $\mathrm{H}_{2}$ evolution is thought to proceed through a sensitizing mechanism initiated by excitation of the surface complex by visible light followed by electron injection into the conduction band of $\mathrm{TiO}_{2}$. Such a surface charge transfer has been studied and has been confirmed for a $\mathrm{TiO}_{2}$-catecholate surface complex system [17]. The injected electrons in the $\mathrm{TiO}_{2}$ particles migrate to the platinum deposits, where reduction of $\mathrm{H}^{+}$takes place to give $\mathrm{H}_{2}$. TEOA must work as a sacrificial electron donor to reduce the oxidized form of the surface 
complex. A schematic model of the overall reaction is shown in Scheme 2. Judging from the wavelength of the $\mathrm{bn}(\mathrm{OH})_{2}$ complex absorption edge, ca. $580 \mathrm{~nm}$ (ca. $2.1 \mathrm{eV}$ ), and the fact that the complex in an excited state can inject electrons into the conduction band of $\mathrm{TiO}_{2}$, the ground-state electronic level of the complex should be ca. $1 \mathrm{~V}$ negative from the upper edge of the valence band in $\mathrm{TiO}_{2}$. Methanol could inject electrons into the ground state, but negligible $\mathrm{H}_{2}$ was liberated as described above. The difference in the adsorption abilities of a bn $(\mathrm{OH})_{2}$-modified surface, but not a bare $\mathrm{TiO}_{2}$ surface, and/or in the reactivity of the oxidized form of $\operatorname{bn}(\mathrm{OH})_{2}$ complex toward TEOA and methanol might cause the distinction of these sacrificial donors.

\section{Conclusions}

The present study has shown that derivatization of a semiconductor surface with a simple aromatic compound as a complexation reagent can induce a visible-light-induced photocatalytic ability. Although a similar photosensitized water reduction system, which includes a surface complex between $\mathrm{TiO}_{2}$ and 8-hydroxylquinoline, has been demonstrated [31], the present system, proved to drive a photocatalytic reaction under visible-light irradiation, can be further extended. In the present study, we used only a racemate of bn $(\mathrm{OH})_{2}$, but when optically active (R)- and (S)-bn(OH) $)_{2}$ 's are used as complexing agents, we can expect stereoselective photocatalytic reactions (of, for example, phenylethylamine having a chiral carbon center, which has been confirmed to work as an electron donor in the present system) through chiral modification. Further studies along this line are now under way.

\section{Acknowledgements}


The authors are grateful to the Catalysis Society of Japan for supplying $\mathrm{TiO}_{2}$ samples. HyCOM $\mathrm{TiO}_{2}$ was a generous gift from Dr. Hiroshi Kominami (Kinki University). This research was partly supported by a Grant-in-Aid for Scientific Research on Priority Areas (No. 14050007) from The Ministry of Education, Culture, Sports, Science and Technology. Financial support by The Sumitomo Foundation is acknowledged. Mr. Tetsuzo Habu and Mr. Kazuhiro Matsudaira (The Technical Division of the Catalysis Research Center, Hokkaido University) are acknowledged for their assistance in the construction of the photoirradiation apparatuses. The authors would also like to thank the staff of the Center for Instrumental Analysis, Hokkaido University for their assistance in elemental analyses.

\section{References}

[1] M. Schiavello (Ed.), Heterogeneous Photocatalysis, John Wiley \& Sons, Chichester, 1997.

[2] A. Fujishima, K. Hashimoto, and T. Watanabe, $\mathrm{TiO}_{2}$ Photocatalysis, Fundamentals and Applications, BKC, Inc., Tokyo, 1999.

[3] W. Choi, A. Termin, M. R. Hoffmann, J. Phys. Chem. 98 (1994) 13669.

[4] M. Anpo, Y. Ichihashi, M. Takeuchi, H. Yamashita, Res. Chem. Intermed. 24 (1998) 143.

[5] K. Takeuchi, I. Nakamura, O. Matsumoto, S. Sugihara, M. Ando, T. Ihara, Chem. Lett. (2000) 1354.

[6] S. Sato, Chem. Phys. Lett. 123 (1986) 126.

[7] R. Asahi, T. Morikawa, T. Ohwaki, K. Aoki, Y. Tada, Science, 293 (2001) 269.

[8] R. Abe, K. Hara, K. Sayama, K. Domen, H. Arakawa, J. Photochem. Photobiol. A: Chem. 137 (2000) 63.

[9] D. Chatterjee, A. Mahata, J. Photochem. Photobiol. A: Chem. 153 (2002) 199. 
[10] A. Hagfeldt, M. Grätzel, Acc. Chem. Res., 33 (2000) 269.

[11] K. Hara, K. Sayama, Y. Ohga, A. Shinpo, S. Suga, H. Arakawa, Chem. Commun. (2001) 569.

[12] J. J. He, G. Benko, F. Korodi, T. Polivka, R. Lomoth, B. Akermark, L. C. Sun, A. Hagfeldt, V. Sundstrom, J. Am. Chem. Soc. 124 (2002) 4922.

[13] P. A. Connor, K. D. Dobson, A. J. McQuillan, Langmuir 11 (1995) 4193.

[14] T. Awatani, K. D. Dobson, A. J. McQuillan, B. Ohtani, K. Uosaki, Chem. Lett. (1998) 849.

[15] B. A. Borgias, S. R. Cooper, Y. B. Koh, K. N. Raymond, Inorg. Chem. 23 (1984) 1009.

[16] R. Rodrigues, M. A. Blesa, A. E. Regazzoni, J. Colloid Interface Sci. 177 (1996) 122.

[17] Y. Liu, J. I. Dadap, D. Zimdars, K. B. Eisenthal, J. Phys. Chem. B. 103 (1999) 2480.

[18] J. Moser, S. Punchihewa, P. P. Infelta, M. Grätzel, Langmuir 7 (1991) 3012.

[19] A. E. Regazzoni, P. Mandelbaum, M. Matsuyoshi, S. Schiller, S. A. Bilmes, M. A. Blesa, Langmuir 14 (1998) 868.

[20] S. Ikeda, C. Abe, T. Torimoto, B. Ohtani, Electrochemistry 70 (2002) 442.

[21] H. Kominami, J.-i. Kato, Y. Takada, B. Ohtani, J.-i. Kawaguchi, M. Kozawa, S.-i. Nishimoto, T. Inui, K. Izawa, J. Chem. Soc. Faraday Trans. 91 (1995) 1103.

[22] H. Kominami, T. Matsuura, K. Inui, B. Ohtani, S.-i. Nishimoto, Y. Kera, Chem. Lett. (1995) 693.

[23] T. Torimoto, N. Nakamura, S. Ikeda, B. Ohtani, Phys. Chem. Chem. Phys. 4 (2002) 5910.

[24] S. Ikeda, N. Sugiyama, B. Pal, G. Marci, L. Palmisano, H. Noguchi, K. Uosaki, B. Ohtani, Phys. Chem. Chem. Phys. 3 (2001) 267.

[25] L. Kavan, M. Grätzel, S. E. Gilbert, C. Klemenz, H. J. Scheel, J. Am. Chem. Soc. 118 (1996) 6716.

[26] B. Ohtani, S.-w. Zhang, S.-i. Nishimoto, T. Kagiya, J. Chem. Soc. Faraday Trans. 88 
(1992) 1049.

[27] S. Sato, T. Kadowaki, Electrochemistry 57 (1989) 1151.

[28] B. Ohtani, Y. Ogawa, S.-i. Nishimoto, J. Phys. Chem. B 101 (1997) 3746.

[29] B. Ohtani, K. Iwai, S.-i. Nishimoto, S. Sato, J. Phys. Chem. B 101 (1997) 3349.

[30] R. Wang, N. Sakai, A. Fujishima, T. Watanabe, K. Hashimoto, J. Phys. Chem. B 103 (1999) 2188.

[31] V. H. Houlding, M. Grätzel, J. Am. Chem. Soc. 105 (1983) 5695. 


\section{Figure and Scheme Captions}

Figure 1. Ultraviolet and visible light DR spectra of (I) P25, (II) TIO-3, (I') bn( $\mathrm{OH})_{2}$-modified P25, and (II’) bn(OH) $)_{2}$-modified TIO-3.

Figure 2. Relations between $\mathrm{M}_{\mathrm{bn}}$ and $\mathrm{I}_{450}$ (upper) or $\mathrm{S}$ (lower) for (a) Degussa P25, (b) Degussa F281, (c) Ishihara ST-01, (d) Ishihara CR-EL, (e) Hombikat UV-100, (f) Showa Denko F-6, (g) Idemitsu UF, (h) TIO-2, (i) TIO-3, and (j) HyCOM. Open circles, filled circles, open triangles, and filled triangles denote rutile, anatase, their mixtures, and amorphous, respectively.

Figure 3. Time courses of $\mathrm{H}_{2}$ liberation by unmodified (open symbols) and bn $(\mathrm{OH})_{2}$-modified (closed symbols) TIO-3 (2 wt\% Pt) suspended in a deaerated aqueous methanol (squares) or TEOA (circles) solution under ultraviolet-light (> $300 \mathrm{~nm}$ ) irradiation.

Figure 4. Time courses of $\mathrm{H}_{2}$ liberation by unmodified (open circles) and bn $(\mathrm{OH})_{2}$-modified (closed circles) TIO-3 (1 wt\% Pt) suspended in a deaerated aqueous TEOA solution under visible-light (> $430 \mathrm{~nm})$ irradiation.

Figure 5. Dependence of rate of $\mathrm{H}_{2}$ liberation by bn(OH)$)_{2}$-modified TIO-3 loaded with 0.5 wt\% of Pt on wavelength of photoirradiated light: (A) > $430 \mathrm{~nm}$, (B) $>490$ $\mathrm{nm},(\mathrm{C})>540 \mathrm{~nm},(\mathrm{D})>580 \mathrm{~nm}$.

Figure 6. Dependence of rate of $\mathrm{H}_{2}$ liberation by bn(OH) $)_{2}$-modified TIO-3 on amount of Pt deposits. Rate of $\mathrm{H}_{2}$ liberation was estimated from the slope of the time course curve in the initial $2 \mathrm{~h}$ of photoirradiation. 
Scheme 1. Schematic illustration of the reactions between Ti-OH and phenolic hydroxyl group in bn $(\mathrm{OH})_{2}$ through (i) chelation (a 1:2 adduct) and (ii) esterification (a 2:2 adduct), both of which are accompanied by the liberation of water.

Scheme 2. Proposed model for overall reaction of visible light-induced photocatalytic $\mathrm{H}_{2}$ evolution by bn $(\mathrm{OH})_{2}$-modified $\mathrm{TiO}_{2}$ loaded with platinum. 


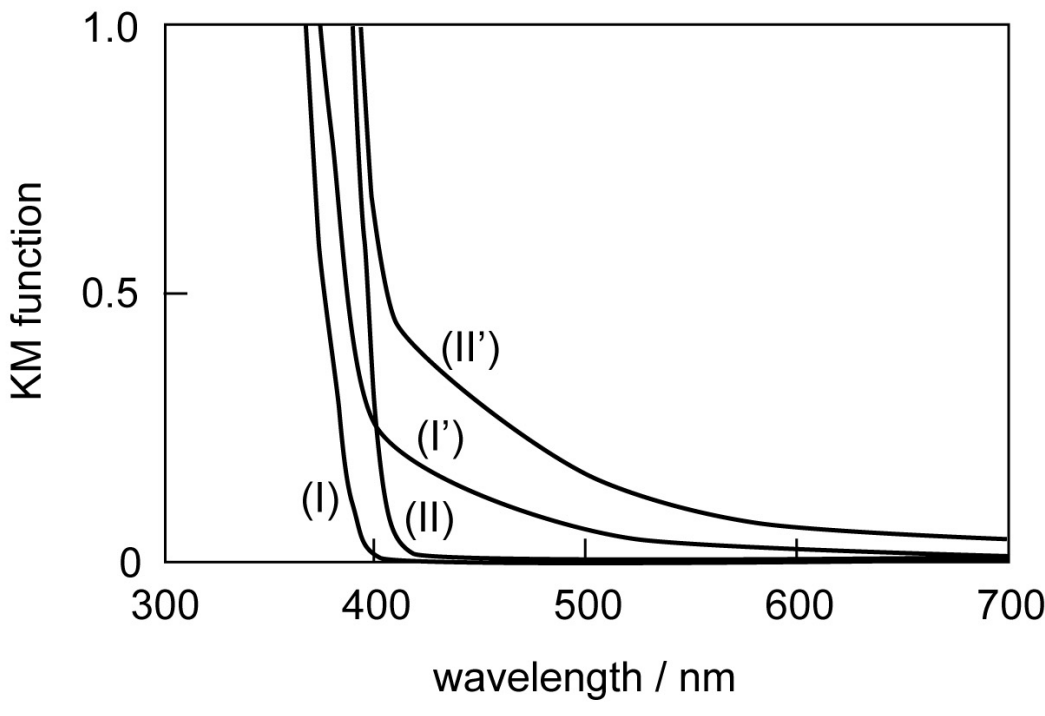

Figure 1. S. Ikeda, C. Abe, T. Torimoto, and B. Ohtani 


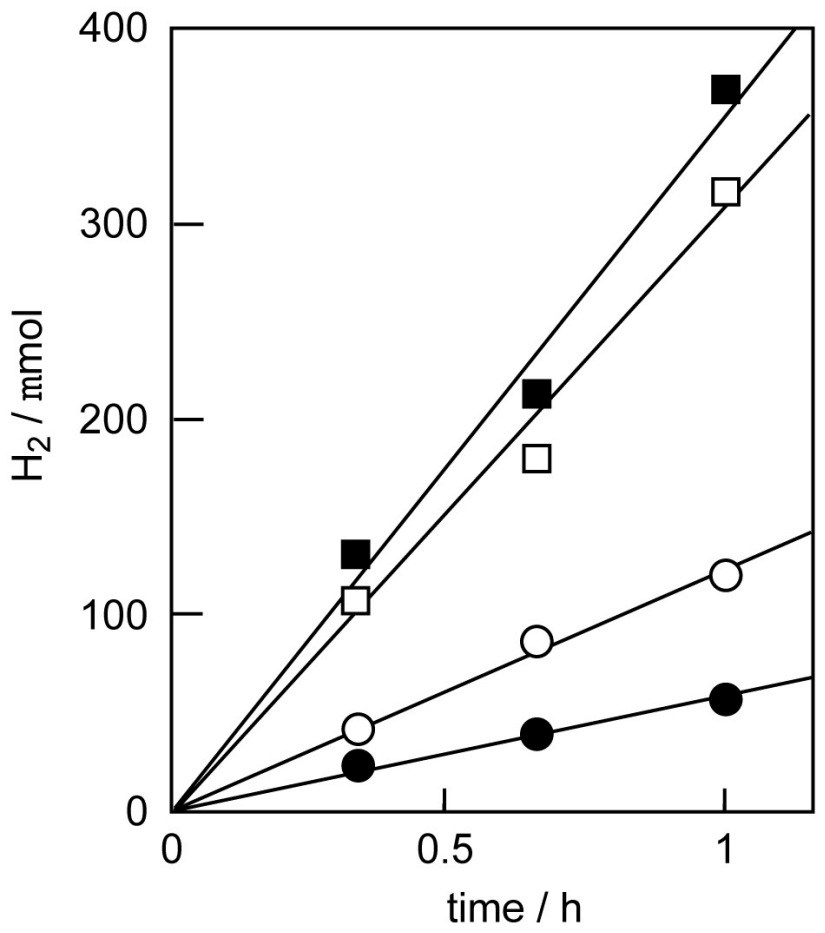

Figure 3. S. Ikeda, C. Abe, T. Torimoto, and B. Ohtani 


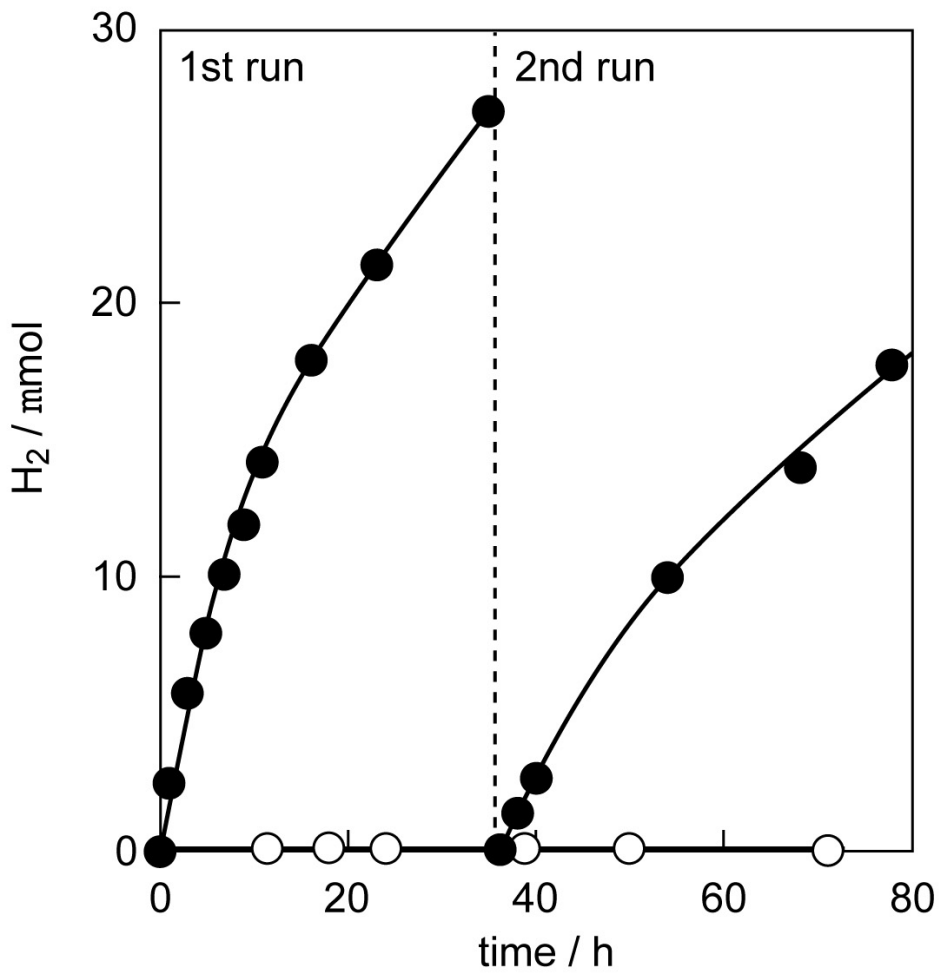

Figure 4. S. Ikeda, C. Abe, T. Torimoto, and B. Ohtani 


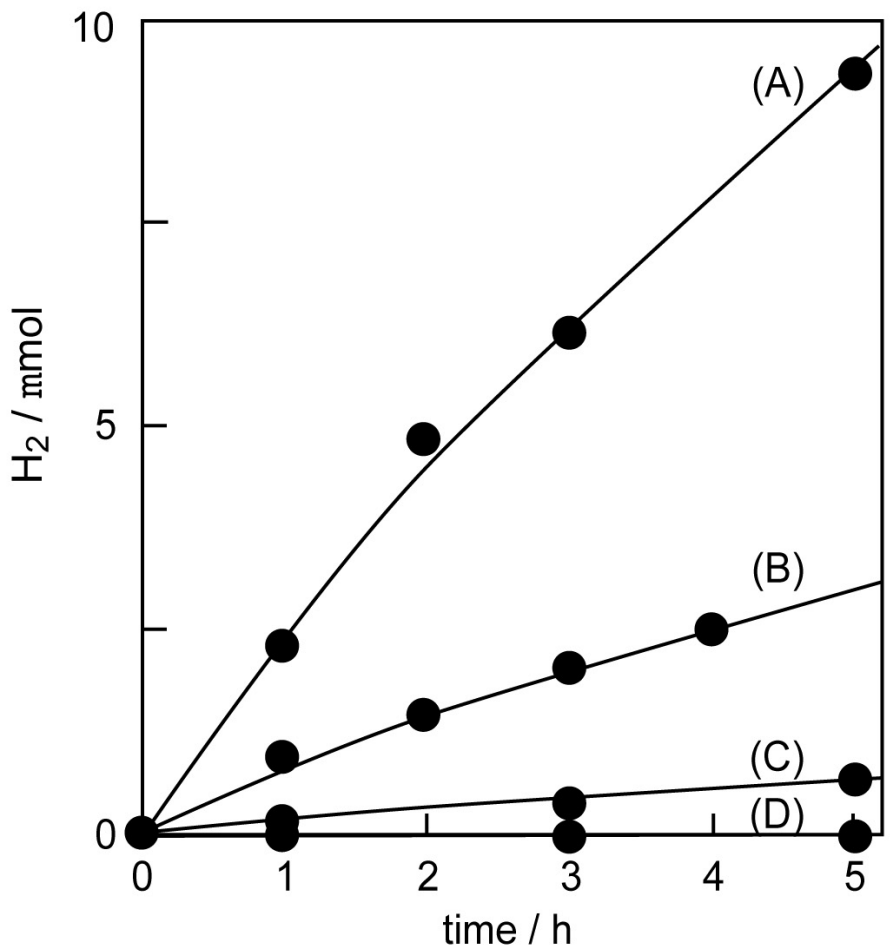

Figure 5. S. Ikeda, C. Abe, T. Torimoto, and B. Ohtani 


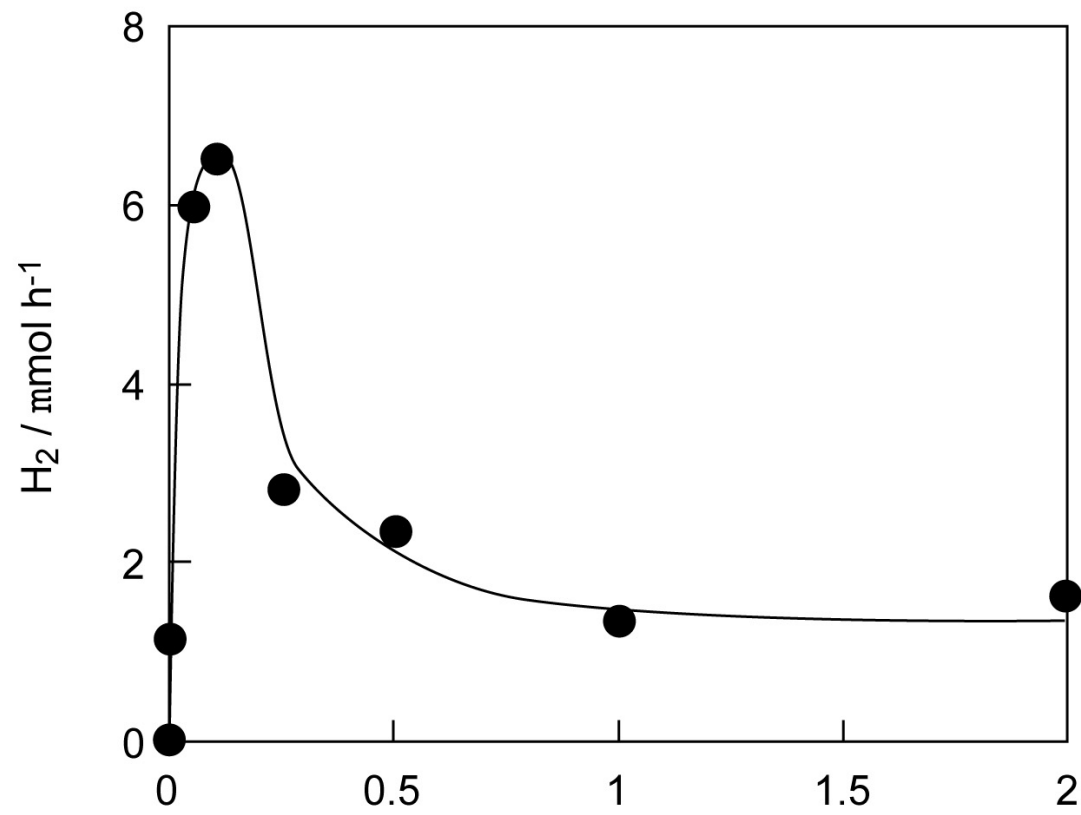

$\mathrm{Pt} / \mathrm{wt} \%$

Figure 6. S. Ikeda, C. Abe, T. Torimoto, and B. Ohtani 